\title{
Breakthrough pain in patients with controlled or uncontrolled pain: An observational study
}

\author{
Antonio Gatti MD¹, Marta Gentili MS², Marco Baciarello MD³, Marzia Lazzari MD¹, Rossella Marzi MD, \\ Elisa Palombo ${ }^{1}$, Alessandro F Sabato $\mathrm{MD}^{1}$, Guido Fanelli $\mathrm{MD}^{3}$
}

\begin{abstract}
A Gatti, M Gentili, M Baciarello, et al. Breakthrough pain in patients with controlled or uncontrolled pain: An observational study. Pain Res Manag 2014;19(6):e168-e171.
\end{abstract}

BACKGROUND: Breakthrough pain (BTP) is traditionally defined as a pain exacerbation in patients with chronic controlled pain. However, this definition has recently been challenged.

OBJECTIVES: To evaluate the prevalence of unsatisfactory control in patients with chronic cancer pain, and investigate the frequency and intensity of BTP episodes.

METHODS: A total of 665 patients with chronic cancer pain attending 21 pain therapy units in Italy were evaluated for baseline pain intensity and number of BTP episodes over a 30-day period. All patients started, continued or modified treatment for BTP at enrollment, according to medical judgment.

RESULTS: The number of BTP events was higher in patients with uncontrolled baseline pain, although the intensity and duration of episodes were similar. In patients with uncontrolled baseline pain, the number of events decreased with time and reached values comparable with those reported in patients with controlled pain. Both the intensity of the pain and the duration of the BTP events exhibited similar values in the two groups at all time points, following increased monitoring and the prescription of analgesic medication.

CONCLUSION: Patients with uncontrolled baseline pain experienced BTP flares with higher frequency, but similar intensity and duration with respect to patients with controlled pain at baseline. Notably, a close followup and adequate management of the BTP episodes led to an improvement of BTP in the observed patients.

Key Words: BTP; Oncological pain; Pain treatment

\section{L'accès douloureux paroxystique chez des patients souffrant de douleur contrôlée ou incontrôlée : une étude d'observation}

\begin{abstract}
HISTORIQUE : D'ordinaire, l'accès douloureux paroxystique (ADP) est défini comme une exacerbation de la douleur chez des patients souffrant de douleur chronique contrôlée. Cependant, cette définition a récemment été remise en question.

OBJECTIFS : Évaluer la prévalence de contrôle insatisfaisant de la douleur cancéreuse chronique chez les patients et examiner la fréquence et l'intensité des ADP.

MÉTHODOLOGIE : Au total, 665 patients atteints de douleur cancéreuse chronique qui ont participé à 21 unités de thérapie contre la douleur en Italie ont été évalués pour déterminer l'intensité de leur douleur de départ et le nombre d'ADP sur une période de 30 jours. Tous les patients ont amorcé, poursuivi ou modifié le traitement de l'ADP en début d'étude, selon le jugement médical.

RÉSULTATS : Le nombre d'ADP était plus élevé chez les patients dont la douleur de départ était incontrôlée, même si la durée et l'intensité des épisodes étaient similaires. Chez les patients dont la douleur de départ était incontrôlée, le nombre d'épisodes a diminué au fil du temps et atteint des valeurs comparables à celles déclarées chez les patients dont la douleur était contrôlée. Tant l'intensité de la douleur que la durée des ADP avaient des valeurs similaires dans les deux groupes à chacun des points dans le temps, après une surveillance accrue et la prescription d'analgésiques.

CONCLUSION : Les patients ayant une douleur de départ incontrôlée souffraient de plus d'ADP, mais leur durée et leur intensité étaient similaires à celles des patients ayant une douleur de départ contrôlée. Notamment, un suivi étroit et une prise en charge pertinente de l'ADP ont suscité une amélioration de l'ADP chez les patients observés.
\end{abstract}

several concurrent factors (13). In oncological patients, the prevalence of BTP ranges from $40 \%$ to $80 \%(14-17)$, while in nononcological patients it is $>55 \%(7,9)$. A better knowledge of the mechanisms underlying BTP and the use of appropriate treatments appears to be necessary to increase the quality of life of patients who experience such sudden exacerbations of pain (12).

The aim of the present prospective observational study was to evaluate the frequency, duration and intensity of BTP episodes in patients with controlled or uncontrolled cancer pain at baseline. We also evaluated the effectiveness of pain treatment in improving the characteristics of BTP.

\section{METHODS}

The present prospective, observational study was conducted in 21 Italian outpatient pain clinics from November 15, 2012 to February 15, 2013. Consecutive patients with chronic cancer pain (Eastern Cooperative Oncology Group performance status 0 to 1 ) were enrolled in the study. Patients who had undergone radiation therapy, cementoplasty or analgesic procedures other than pharmacological treatments the characteristics of patients in whom painful flares can be defined as $\operatorname{BTP}(7)$.

BTP is observed in oncological and nononcological patients (8-11), and also in patients without background pain (12). Epidemiological data showed that BTP has a high prevalence in the population with very severe background pain $(8,9,11)$, and BTP has been associated with

\footnotetext{
${ }^{1}$ Emergency Care, Critical Care Medicine, Pain Medicine and Anesthesiology Department, Tor Vergata Polyclinic, University of Rome Tor

Vergata, Rome; ${ }^{2}$ Patient association "Vivere senza dolore", Milan; ${ }^{3}$ Anesthesia, Critical Care and Pain Medicine Unit, Department of Surgical

Sciences, University of Parma, Parma; ${ }^{4}$ Pain Therapy Department Maggiore della carità Hospital, Novara, Italy

Correspondence: Marta Gentili, Patient association "Vivere senza dolore", Piazza E L Morselli 1, 20154 Milan, Italy. Telephone 39-02-313835, fax 39-02-49542408, e-mail presidenza@viveresenzadolore.it
} 
TABLE 1

Characteristics of the patients entering the study

\begin{tabular}{lcc}
\hline & \multicolumn{2}{c}{ Baseline pain } \\
\cline { 2 - 3 } Characteristic & Controlled & Uncontrolled \\
\hline Patients, $n$ & 116 & 549 \\
Female & $51(43.9)$ & $262(47.8)$ \\
Male & $65(56.1)$ & $287(52.2)$ \\
Age, years, & $66.9 \pm 15.8$ & $63.7 \pm 13.8$ \\
$\quad$ mean \pm SD & & \\
NRS score, mean \pm SD & $2.4 \pm 0.6$ & $6.9 \pm 1.5$ \\
\hline
\end{tabular}

Data presented as $n$ (\%) unless otherwise indicated. NRS Numerical rating scale

were not eligible. Written informed consent to participate was obtained from each subject before the enrollment. The study was approved by the ethics committees of the participating clinics. All data were collected using a dedicated questionnaire.

Patient data are presented according to their background pain intensity (average pain in the seven days before enrollment, according to patient recall), which was evaluated using an 11-point numerical rating scale (NRS) ranging from 0 (no pain) to 10 (worst imaginable pain). The first group consisted of patients with controlled background pain (NRS $\leq 3)$, whereas the second group included patients with uncontrolled pain (NRS scale $>3$ ). The NRS cut-off of 3 was chosen according to WHO guidelines (18).

Pain intensity in all patients was monitored on the day of enrollment (T0), after 10 days (T1) and after 30 days (T2), and was measured using an NRS. The occurrence of pain exacerbations, their daily frequency, their mean intensity and their mean duration in minutes were evaluated at T0, T1 and T2. BTP events were defined as rapid, transitory exacerbations of pain distinguishable in intensity from the background pain, irrespective from the control of baseline pain, as reported by each patient and lasting between $30 \mathrm{~min}$ and $40 \mathrm{~min}$. All patients continued their standard therapy for background pain, if any, and current medications were recorded at each time point. All patients started, continued or modified treatment for BTP at TO, according to the clinical judgment of their treating physicians.

All data were analysed by descriptive statistics. P values were twosided and were determined using a repeated-measures ANOVA; $\mathrm{P}<0.05$ was considered to be statistically significant.

\section{RESULTS}

A total of 665 patients provided consent and were considered for the subsequent statistical analysis. All patients completed the study. Patients characteristics are summarized in Table 1. A total of 116 patients had controlled background pain, whereas 549 patients were referred to participating pain clinics with poorly controlled background pain.

\section{Pain parameters}

Table 2 presents the different pain parameters in the two groups at the different time points.

At baseline, the mean $( \pm$ SD) NRS value was $2.4 \pm 0.6$ in the controlled-pain population and $6.9 \pm 1.5$ in patients with uncontrolled background pain $(\mathrm{P}<0.05)$. The number of BTP was $2.6 \pm 1.3$ in patients with controlled pain and $3.5 \pm 1.5$ in those with uncontrolled pain $(\mathrm{P}<0.05)$, and the BTP intensity was $6.2 \pm 2.4$ and $8.1 \pm 1.8$ $(\mathrm{P}<0.05)$, respectively. Mean duration of exacerbation pain was $21.2 \pm 12.5 \mathrm{~min}$ and $25.2 \pm 11.8 \mathrm{~min}$ in patients with controlled and uncontrolled background pain, respectively.

The intensity of the background pain did not decrease throughout the study in patients with controlled background pain at baseline (T1: $2.7 \pm 1.1 ; \mathrm{T} 2: 2.5 \pm 1.0$, respectively). Similarly, in these patients, the number of BTP and the mean intensity of pain exacerbations were similar to those reported at baseline. However, the mean duration of episodes of BTP was significantly lower at T1 (12.0 \pm 8.9 min compared with $21.2 \pm 12.5 \mathrm{~min}$ at $\mathrm{TO} ; \mathrm{P}<0.0001)$; the observed decrease in pain duration persisted at T2 $(\mathrm{P}<0.0001$ versus baseline $)$.

In patients with uncontrolled background pain at baseline, NRS of background pain was significantly reduced at $\mathrm{T} 1$ and $\mathrm{T} 2$ (T1: $5.1 \pm 1.8$, $\mathrm{P}<0.0001$ versus T0; T2: $3.8 \pm 1.4, \mathrm{P}<0.0001$ versus $\mathrm{T} 0$ and $\mathrm{T} 1)$ respectively. In addition, a significant reduction in the number, intensity and duration of the pain exacerbations was recorded at both time points.

The comparison between the two groups at T1 and T2 did not reveal any significant difference in the number of BTP events. In addition, both the intensity of the pain and the duration of the BTP episodes in the two groups were similar at T1 and T2.

\section{Pharmacological treatment}

Table 3 presents the analgesic treatments prescribed for the control of background pain in each group at different timepoints, while Table 4 presents the drugs used for the treatment of BTP.

At baseline, the main drugs for background pain treatment in patients with controlled background pain were: fentanyl transdermal therapeutic system $(n=32$ [27.5\%]); prolonged-release oxycodone/ naloxone or nonsteroidal anti-inflammatory drugs (NSAIDs) $(\mathrm{n}=18$ [15.5\%] each); and controlled-release oxycodone controlled release or acetaminophen ( $n=9$ [7.8\%] each). The most commonly prescribed drugs for exacerbations of pain were buccal fentanyl tablets in 28 patients (24.1\%); transmucosal fentanyl in 24 (20.7\%); NSAIDs in $16(13.8 \%)$; and oral morphine instant release in $13(11.2 \%)$. Twenty patients $(17.2 \%)$ were not under any treatment plan for BTP episodes. In patients with uncontrolled background pain at baseline, 154 patients (28\%) of patients were not taking any therapy for BTP at the time of enrollment. In addition, 10 patients $(1.8 \%)$ were not taking any analgesic therapy, despite the presence of uncontrolled pain. A substantial increase from $\mathrm{T} 0$ to $\mathrm{T} 2$ in the use of prolonged-release oxycodone/naloxone prolonged release (from 83 [15.1\%] patients to $124[22.6 \%]$ patients) and a significant reduction in the use of NSAID (from $53[9.6 \%]$ patients to 19 [3.5\%] patients), tramadol (from 47 [8.6\%] patients to 13 [2.4\%] patients) and codeine/acetaminophen (from $25[4.4 \%]$ patients to $6[1.1 \%]$ patients) were reported. With regard to the drugs used for the treatment of pain exacerbations, an increase in the prescription of fentanyl from T0 to T2 was reported in the different formulations (buccal tablets, from 91 [16.6\%] patients to 187 [34.1\%] patients; transmucosal, from 59 [10.7\%] patients to 142 [25.8\%] patients; and nasal spray with pectin, from 19 [3.5\%] patients to $66[12.0 \%]$ patients). Conversely, a marked reduction in the use of NSAIDs (from 105 [19.1\%] patients to 26 [4.7\%] patients) and immediate-release morphine (from 76 [13.8\%] patients to $26[4.7 \%]$ patients) was observed. The percentage of patients not receiving any treatment for BTP episodes at T0 decreased form $28.1 \%$ to $0 \%$.

In patients with controlled pain at baseline, mean morphine equivalent daily doses went from $120.75 \pm 28.30 \mathrm{mg}$ at T0 to $136.52 \pm 31.84 \mathrm{mg}$ at $\mathrm{T} 1$ and $147.26 \pm 120.27 \mathrm{mg}$ at $\mathrm{T} 2(\mathrm{P}<0.05$ for $\mathrm{T} 1$ and T2 versus TO) (equivalence according to Hanks et al [19]). On the other hand, in patients with uncontrolled pain at baseline, morphine equivalent daily doses went from $93.07 \pm 113.16 \mathrm{mg}$ at T0 to $109.73 \pm 56.65 \mathrm{mg}$ at $\mathrm{T} 1$ and $127.66 \pm 58.7 \mathrm{mg}$ at $\mathrm{T} 2(\mathrm{P}<0.05$ for $\mathrm{T} 2$ versus $\mathrm{TO}$ ).

\section{DISCUSSION}

Although historically defined as a pain exacerbation in patients with chronic controlled pain, either oncological or nononcological in nature $(1,2)$, BTP has recently been reported to occur in patients without chronic pain as well (12). A precise definition of BTP is still under debate, and the patients in whom exacerbation of pain can be classified as BTP have not been clearly identified. Oncological patients who experience BTP show increased anxiety and depression and, more generally, a decreased satisfaction with the therapy $(11,20-23)$. This also affects the family of the patient, as well as physicians, caregivers and, more broadly, the health system $(19,23$. $25)$. On the above-mentioned basis, a proper management of BTP is 


\begin{tabular}{|c|c|c|c|c|c|c|}
\hline & \multicolumn{6}{|c|}{ Baseline pain } \\
\hline & \multicolumn{3}{|c|}{ Controlled } & \multicolumn{3}{|c|}{ Uncontrolled } \\
\hline Baseline numerical rating scale score & $2.4 \pm 0.6$ & $2.7 \pm 1.1$ & $2.5 \pm 1.0$ & $6.9 \pm 1.5^{*}$ & $5.1 \pm 1.8^{\dagger}$ & $3.8 \pm 1.4^{\dagger \ddagger}$ \\
\hline Mean numerical rating scale score for intensity & $6.2 \pm 2.4$ & $6.0 \pm 2.2$ & $5.7 \pm 2.3$ & $8.1 \pm 1.8^{*}$ & $7.0 \pm 1.9^{\dagger}$ & $6.2 \pm 1.9^{\dagger}$ \\
\hline Mean duration of the pain exacerbation, min & $21.2 \pm 12.5$ & $12.0 \pm 8.9^{\dagger}$ & $11.7 \pm 8.9^{\dagger}$ & $25.2 \pm 11.8$ & $14.4 \pm 9.6^{\dagger}$ & $11.7 \pm 8.8^{\dagger}$ \\
\hline
\end{tabular}

Data presented as mean $\pm S D$; ${ }^{*}<<0.05$ versus patients with controlled baseline pain; ${ }^{t} P<0.0001$ versus $T 0 ; \ddagger P<0.0001$ versus T1. BTP Breakthrough pain. T0 Time of enrollment; $T 110$ days after enrollment; T2 30 days after enrollment

TABLE 3

Analgesic treatments prescribed for the control of background pain

\begin{tabular}{|c|c|c|c|}
\hline & TO & T1 & T2 \\
\hline \multicolumn{4}{|c|}{ Controlled background pain $(n=116)$} \\
\hline None & 5.0 & 3.7 & 2.4 \\
\hline NSAIDs & 15.7 & 16.3 & 16.7 \\
\hline Acetaminophen & 7.9 & 8.9 & 11.1 \\
\hline Tramadol & 2.1 & 0.7 & 0.8 \\
\hline Codeine/acetaminophen & 0.7 & 1.5 & 0.0 \\
\hline Tapentadol PR & 2.9 & 2.2 & 2.4 \\
\hline Morphine CR & 6.4 & 10.4 & 7.9 \\
\hline Oxycodone/acetaminophen IR & 2.9 & 3.0 & 1.6 \\
\hline Oxycodone CR & 7.9 & 7.4 & 9.5 \\
\hline Oxycodone/naloxone PR & 15.7 & 14.8 & 14.3 \\
\hline Hydromorphone CR & 3.6 & 3.0 & 2.4 \\
\hline Fentanyl TTS & 27.1 & 27.4 & 31.0 \\
\hline Buprenorphine TTS & 2.1 & 0.7 & 0.0 \\
\hline \multicolumn{4}{|c|}{ Uncontrolled background pain $(n=549)$} \\
\hline None & 1.8 & 0.2 & 0.3 \\
\hline NSAIDs & 9.6 & 3.1 & 3.4 \\
\hline Acetaminophen & 13.5 & 13.1 & 13.1 \\
\hline Tramadol & 8.5 & 2.3 & 2.3 \\
\hline Tramadolo/acetaminophen & 1.2 & 0.0 & 0.0 \\
\hline Codeine & 0.4 & 0.5 & 0.3 \\
\hline Codeine/acetaminophen & 4.5 & 1.0 & 1.0 \\
\hline Tapentadol PR & 2.1 & 2.4 & 2.1 \\
\hline Morphine CR & 5.8 & 6.7 & 6.7 \\
\hline Oxycodone/acetaminophen IR & 2.8 & 2.3 & 3.1 \\
\hline Oxycodone CR & 8.1 & 8.6 & 8.8 \\
\hline Oxycodone/naloxone PR & 15.2 & 23.5 & 22.6 \\
\hline Methadone & 0.3 & 0.5 & 0.5 \\
\hline Hydromorphone CR & 4.8 & 9.8 & 11.0 \\
\hline Fentanyl TTS & 19.3 & 22.2 & 21.3 \\
\hline Buprenorphine TTS & 2.1 & 3.9 & 3.4 \\
\hline
\end{tabular}

Data presented as \%. CR Controlled release; IR Immediate release; NSAID Nonsteroidal anti-inflammatory drug; PR Prolonged release; TO Time of enrollment; T1 10 days after enrollment; T2 30 days after enrollment; TTS Transdermal therapeutic system

a unmet medical need that has increasingly gained attention in the past several years.

Our study aimed to investigate the occurrence and characteristics of BTP episodes in two populations of oncological patients: subjects with and without a control of background pain at baseline. Of note, baseline pain was controlled in only $<20 \%$ of patients presenting to pain clinics, while, in the majority of patients, pain was not adequately controlled. In addition, some patients were not under any chronic pain treatment plan despite the presence of uncontrolled severe pain. A high proportion of patients was not undergoing any specific treatment for BTP. Collectively, these findings may suggest
TABLE 4

Analgesic treatments prescribed for the control of breakthrough pain

\begin{tabular}{lrrr}
\hline & T0 & T1 & T2 \\
\hline Controlled background pain (n=116) & & & \\
None & 17.5 & 0 & 0 \\
NSAIDs & 13.5 & 17.9 & 15.0 \\
Morphine IR & 11.1 & 12.0 & 7.5 \\
Morphine PCA & 1.6 & 1.7 & 1.9 \\
Fentanyl sublingual & 3.2 & 3.4 & 3.7 \\
Fentanyl transmucosal & 20.6 & 26.5 & 28.0 \\
Fentanyl nasal spray hidrosolubiles & 2.4 & 2.6 & 1.9 \\
Fentanyl buccal tables & 24.6 & 28.2 & 33.6 \\
Fentanyl nasal spray pectina & 5.6 & 7.7 & 8.4 \\
Uncontrolled background pain ( $\mathbf{n}=549)$ & & \\
None & 28.0 & 0 & 0 \\
NSAIDs & 19.1 & 4.9 & 4.9 \\
Morphine IR & 13.8 & 7.7 & 6.0 \\
Morphine PCA & 1.4 & 0.9 & 1.1 \\
Fentanyl sublingual & 4.8 & 11.2 & 10.8 \\
Fentanyl transmucosal & 10.8 & 25.0 & 25.9 \\
Fentanyl nasal spray hidrosolubiles & 2.3 & 5.6 & 5.2 \\
Fentanyl buccal tables & 16.5 & 31.8 & 34.0 \\
Fentanyl nasal spray pectina & 3.4 & 12.9 & 12.1 \\
\hline
\end{tabular}

Data presented as \%. IR Immediate release; NSAID Nonsteroidal antiinflammatory drug; PCA Patient-controlled analgesia; TO Time of enrollment; T1 10 days after enrollment; T2 30 days after enrollment

that the analgesic procedures adopted in clinical practice in the observed population of cancer patients are still suboptimal and require improvement.

While some studies reported peaks of pain intensity independently on pain treatment at baseline, other studies have shown no clear distinction between background and BTP intensity $(14,26)$. Our data show that in patients with both controlled and uncontrolled baseline pain, the increased monitoring and the prescription of treatments for BTP significantly reduced the number, intensity and duration of BTP episodes. Of note, the reduction in the duration of BTP episodes after institution of specific pharmacological management has particular relevance and has not, to our knowledge, been clearly demonstrated before. In both groups, improved monitoring and treatment of BTP was associated with an approximate 50\% reduction in the duration of pain flares. However, despite a significant improvement in the symptoms, with a $50 \%$ reduction in the intensity of pain, at 30 days several patients still experienced uncontrolled pain.

The intensity of background pain, the background analgesic treatment, the intensity of BTP and their response to treatment have rarely been concomitantly assessed in epidemiological and clinical studies of BTP. Recently, Mercadante et al (27) reported the prevalence of BTP in a population of 265 patients, of whom 49 were under suboptimal background analgesia and required optimization of the analgesic treatment. Although the overall prevalence of BTP did not change with 
optimization of the therapy, there was an improvement in the intensity of background pain, and a decrease in the number of episodes per day of BTP with a decreased intensity and duration. The data we report here are similar, but with two major differences. First, our results were obtained in a much larger cohort of patients (549 versus 49), further corroborating the fact that BTP episodes are independent of the pain control at baseline. Second, we evaluated the reduction of BTP events after the prescription of a specific treatment for BTP. Of note, the results showed that better pain management led to a reduction in the duration of BTP episodes.

The findings of the present study may be limited by the fact that 21 unrelated Italian pain clinics provided data; whereas there are obvious disadvantages in terms of lack of homogeneity of therapeutic choices, we believe the larger numbers improve the reliability of our population sample.

Moreover, no multivariate analysis was performed to take into account the possible effect of any confounding factor on the results. However, this analysis would have been hampered by the considerable heterogeneity of the patients and the overall limited sample size. For the same reasons, it was not possible to compare the effectiveness of the different treatments prescribed.

\section{REFERENCES}

1. Portenoy RK, Hagen NA. Breakthrough pain: Definition and management. Oncology (Williston Park) 1989;3:25-9.

2. Portenoy RK, Hagen NA. Breakthrough pain: Definition, prevalence and characteristics. Pain 1990;41:273-81.

3. Davies AN, Dickman A, Reid C, Stevens AM, Zeppetella G. The management of cancer-related breakthrough pain: Recommendations of a task group of the Science Committee of the Association for Palliative Medicine of Great Britain and Ireland. Eur J Pain 2009;13:331-8.

4. Zeppetella G. Impact and management of breakthrough pain in cancer. Curr Opin Support Palliat Care 2009;3:1-6.

5. Mercadante S, Radbruch L, Caraceni A, et al. Episodic (breakthrough) pain: Consensus conference of an expert working group of the European Association for Palliative Care. Cancer 2002;94:832-9.

6. Haugen DF, Hjermstad MJ, Hagen N, Caraceni A, Kaasa S. Assessment and classification of cancer breakthrough pain: A systematic literature review. Pain 2010;149:476-82.

7. Smith H. A comprehensive review of rapid-onset opioids for breakthrough pain. CNS Drugs 2012;26:509-35.

8. Gatti A, Mediati RD, Reale C, et al. Breakthrough pain in patients referred to pain clinics: The Italian pain network retrospective study. Adv Ther 2012;29:464-72.

9. Portenoy RK, Bennett DS, Rauck R, et al. Prevalence and characteristics of breakthrough pain in opioid-treated patients with chronic noncancer pain. J Pain 2006;7:583-91.

10. Portenoy RK, Bruns D, Shoemaker B, Shoemaker SA. Breakthrough pain in community-dwelling patients with cancer pain and noncancer pain, part 2: Impact on function, mood, and quality of life. J Opioid Manag 2010;6:109-16.

11. Portenoy RK, Bruns D, Shoemaker B, Shoemaker SA. Breakthrough pain in community-dwelling patients with cancer pain and noncancer pain, part 1: Prevalence and characteristics. J Opioid Manag 2010;6:97-108.

12. Gatti A, Gentili M, Iorno V, et al. Beyond the traditional definition of breakthrough pain: An observational study. Adv Ther 2013;30:298-305.

13. Davies A, Buchanan A, Zeppetella G, et al. Breakthrough cancer pain: An observational study of 1000 European oncology patients. J Pain Symptom Manage 2013;46:619-28.

14. Caraceni A, Bertetto O, Labianca R, et al. Episodic (breakthrough) pain prevalence in a population of cancer pain patients.

\section{CONCLUSION}

Our study shows that most Italian oncology patients presenting to pain therapy units are experiencing uncontrolled pain at baseline, suggesting the need for better management of this condition. In addition, our data show that even in patients with uncontrolled baseline pain, flares of BTP with intensity and duration similar to those observed in patients with controlled pain are observed, suggesting that poorly managed pain is not a risk factor for higher intensity and number of BTP episodes. Collectively, the results of the present observational study may pave the way for reconsideration of the definition of BTP. We speculate that our data may lead to a new, broader definition of BTP, which should also include patients with uncontrolled baseline pain.

Finally, and of major importance, strict monitoring and adequate management of BTP episodes with the prescription of appropriate drugs can lead to an improvement in the number and intensity of BTP episodes and, notably, the duration of pain flares in oncological patients.

ACKNOWLEDGEMENTS: The authors thank Luca Giacomelli PhD for useful critique.

FUNDING: No grant was used to perform this work.

Comparison of clinical diagnoses with the QUDEI - Italian questionnaire for intense episodic pain. J Pain Symptom Manage 2012:43:833-41.

15. Greco MT, Corli O, Montanari M, Deandrea S, Zagonel V, Apolone G. Epidemiology and pattern of care of breakthrough cancer pain in a longitudinal sample of cancer patients: Results from the Cancer Pain Outcome Research Study Group. Clin J Pain 2011;27:9-18.

16. Margarit C, Julia J, Lopez R, et al. Breakthrough cancer pain - still a challenge. J Pain Res 2012;5:559-66.

17. Webster LR. Breakthrough pain in the management of chronic persistent pain syndromes. Am J Manag Care 2008;14:S116-22.

18. Schug SA, Zech D, Dorr U. Cancer pain management according to WHO analgesic guidelines. J Pain Symptom Manage 1990;5:27-32.

19. Hanks GW, Conno F, Cherny N, et al; Expert Working Group of the Research Network of the European Association for Palliative Care. Morphine and alternative opioids in cancer pain: The EAPC recommendations. Br J Cancer 2001;84:587-93.

20. Burton B, Zeppetella G. Assessing the impact of breakthrough cancer pain. Br J Nurs 2011;20:S14, S6-9.

21. Dickman A. Integrated strategies for the successful management of breakthrough cancer pain. Curr Opin Support Palliat Care 2011;5:8-14.

22. McMillan C. Breakthrough pain: Assessment and management in cancer patients. Br J Nurs 2001;10:860-6.

23. Smith HS. Considerations in selecting rapid-onset opioids for the management of breakthrough pain. J Pain Res 2013;6:189-200.

24. Abernethy AP, Wheeler JL, Fortner BV. A health economic model of breakthrough pain. Am J Manag Care 2008;14:S129-40.

25. Fortner BV, Okon TA, Portenoy RK. A survey of pain-related hospitalizations, emergency department visits, and physician office visits reported by cancer patients with and without history of breakthrough pain. J Pain 2002;3:38-44.

26. Gomez-Batiste X, Madrid F, Moreno F, et al. Breakthrough cancer pain: Prevalence and characteristics in patients in Catalonia, Spain. J Pain Symptom Manage 2002;24:45-52.

27. Mercadante S, Valle A, Porzio G, et al. Relationship between background cancer pain, breakthrough pain, and analgesic treatment: A preliminary study for a better interpretation of epidemiological and clinical studies. Curr Med Res Opin 2013;29:667-71. 


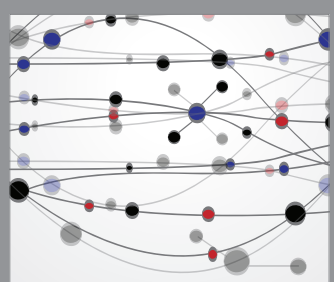

The Scientific World Journal
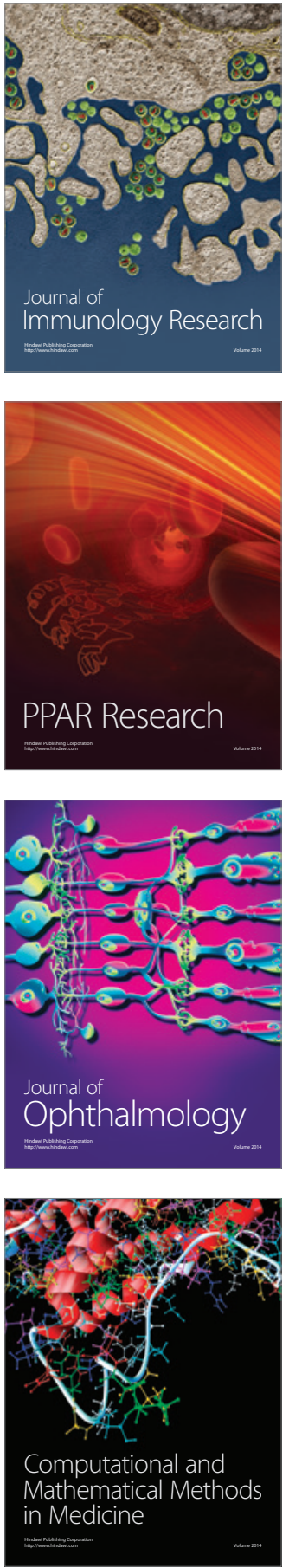

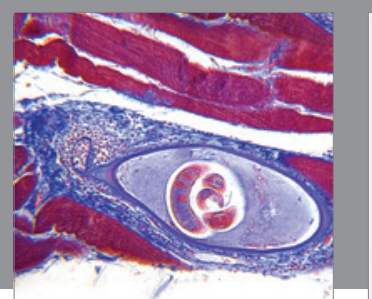

Gastroenterology Research and Practice

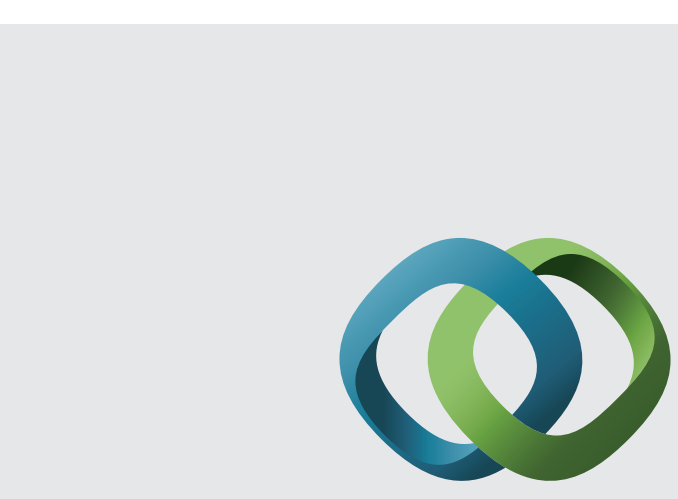

\section{Hindawi}

Submit your manuscripts at

http://www.hindawi.com
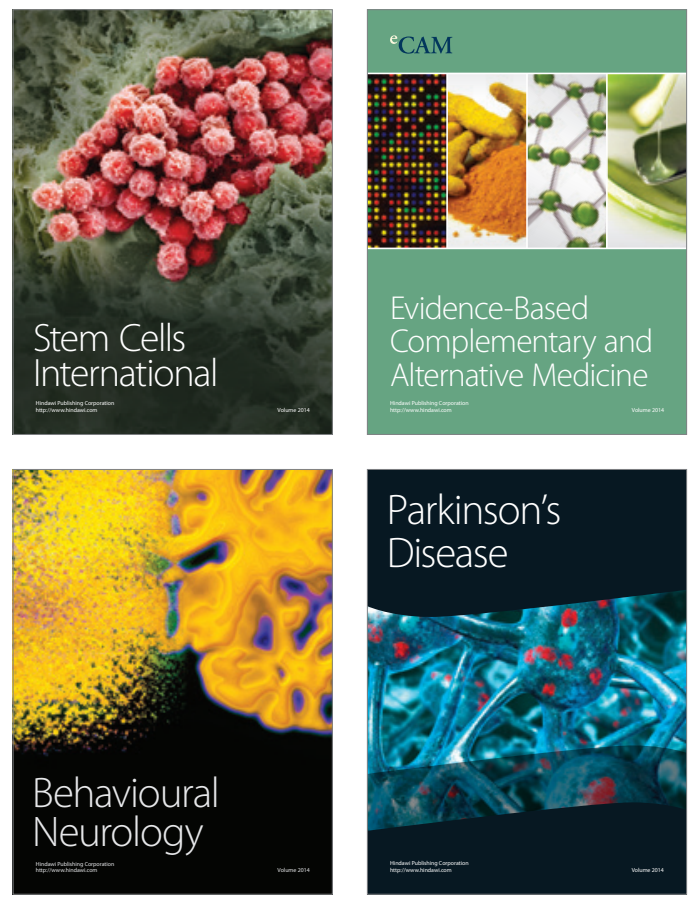
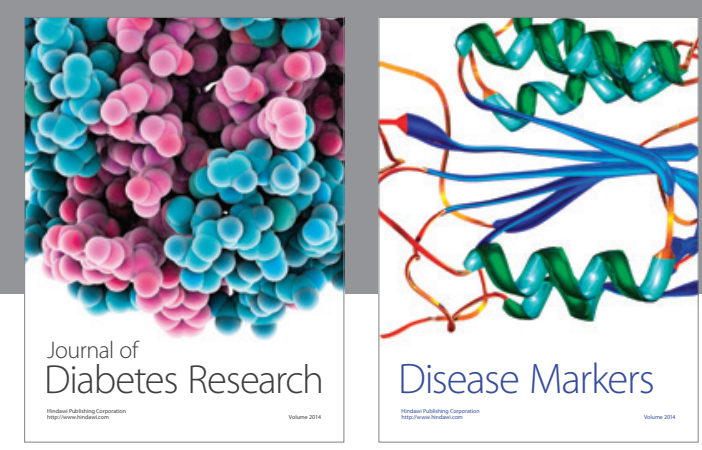

Disease Markers
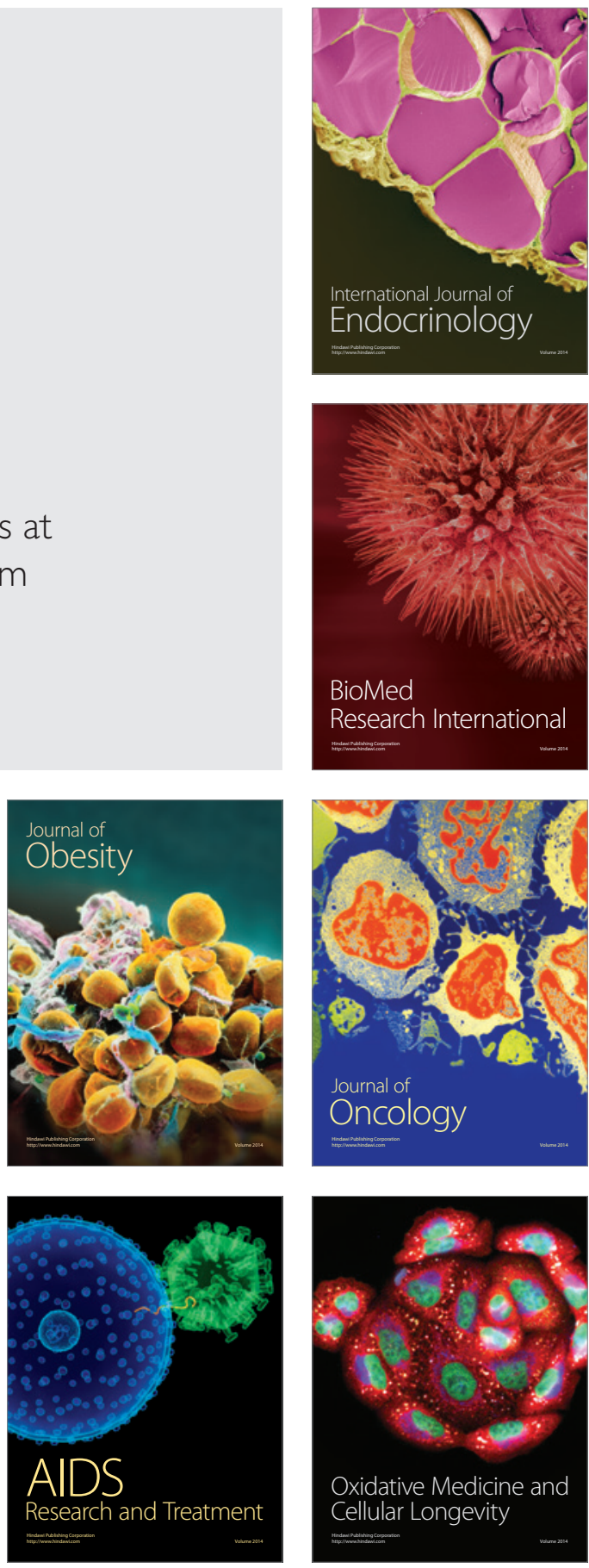